\title{
APPLICATIONS OF THE JOINT ANGULAR FIELD OF VALUES
}

\author{
GEORGE PHILLIP BARKER
}

\begin{abstract}
Let $A_{1}, \ldots, A_{m}$ be $n \times n$ hermitian matrices and let $\nvdash_{n}$ be the real space of $n \times n$ hermitian matrices. If $\operatorname{span}\left\{A_{1}, \ldots, A_{m}\right\}=\mathscr{H}_{n}$, then the extreme rays of the joint angular field of values of $\left\{A_{1}, \ldots, A_{m}\right\}$ are determined. Then this cone is used to give necessary and sufficient conditions for the existence of hermitian matrices $B_{1}, \ldots, B_{m}$ such that $A_{1} \otimes B_{1}+\cdots+A_{m} \otimes B_{m}$ preserves the cone of the positive semidefinite matrices where $A \otimes B$ is the dyad product $A \otimes B(H)=(\operatorname{tr} B H) A$.
\end{abstract}

1. Introduction. Let $V$ be a finite-dimensional real vector space of dimension $n$ with an inner product ( , ). A subset $K \subset V$ is called a (convex) cone iff for all $\alpha, \beta \geq \dot{0}$ and $x, y \in K$ we have $\alpha x+\beta y \in K$. We call $K$ closed if it is a closed set in the natural topology of $V$. The cone $K$ is pointed iff $K \cap(-K)=\{0\}$ (i.e., $K$ contains no nonzero subspace) and full iff $K$ has nonempty interior. In finitedimensional spaces it is well known that $K$ is full iff $K-K=V$. A face $F$ of a cone $K$ is a subcone such that $x, y-x \in K$ and $y \in F$ implies $x \in F$. The dimension of a face $F, \operatorname{dim} F$, is the dimension of the linear span of $F$. If $\operatorname{dim} F=1$ we call $F$ an extreme ray of $K$. If $S \subset K$, then $\phi(S)$ denotes the least face (with respect to inclusion) containing $S$. When $S=\{x\}$, we write simply $\phi(x)$, and if $\phi(x)$ is an extreme ray we call $x$ an extremal.

With any cone $K$ we associate two special cones (cf. [8, Chapter 7]). Set

$$
K^{\prime}=\{y \in V:(y, x) \geq 0, \forall x \in K\} .
$$

Next if $\operatorname{Hom}(V)$ denotes the vector space of linear mappings of $V$, then define

$$
\Pi(K)=\{A \in \operatorname{Hom}(V): A K \subset K\} .
$$

Let $M_{n}(\mathbf{C})$ denote the vector space of $n \times n$ matrices with complex entries and $\mathrm{C}^{n}$ the space of column vectors. Denote by $A^{*}$ or $x^{*}$ the conjugate transpose of $A$ or $x . M_{n}(\mathbf{C})$ is an inner product space under $(A, B)=\operatorname{tr} B^{*} A$. If $A$ and $B$ are hermitian matrices, then $(A, B)$ is real. Thus the set $\mathcal{H}_{n}$ of hermitian matrices is a real inner product space. The set $P_{n}$ of positive semidefinite matrices is a closed pointed full cone in $\mathscr{H}_{n}[\mathbf{1}]$. In fact, $P_{n}$ is self-dual; that is, $P_{n}^{\prime}=P_{n}$, and the faces of $P_{n}$ are known $[\mathbf{1}, \mathbf{3}]$. In particular, the extreme rays are the faces determined by rank one orthogonal projections. The identity matrix $I$ is in the interior of $P_{n}^{\prime}=P_{n}$ so that

$$
B=\left\{P \in P_{n}: \operatorname{tr} P=1=(I, P)\right\}
$$

Received by the editors April 13, 1983 and, in revised form, September 28, 1983.

1980 Mathematics Subject Classification. Primary 15A57, 47D20, 52A20.

Key words and phrases. Numerical range, positive semidefinite matrices, linear transformations of hermitian matrices. 
is a compact convex cross section of $P_{n}$. That is, $B$ is a compact convex subset of $P_{n}$ and for every nonzero $P \in P_{n}$ there is a unique $\alpha>0$ such that $\alpha P \in B$.

An as yet unsolved problem is to determine the set of all linear transformations of $\nvdash_{n}$ which leave $P_{n}$ invariant, that is, describe $\Pi\left(P_{n}\right)$ (cf. [5 and 6]). Work on this problem led to an extension of the classical field of values for a single complex matrix to a kind of joint field of values for a set of complex matrices (cf. [2] and $\S 3)$. In the application we shall take the matrices to be hermitian, but this is not necessary (cf. §2).

Recall that the classical (Hausdorff-Topelitz) field of values for $A \in M_{n}(\mathbf{C})$ is the set

$$
W(A)=\left\{x^{*} A x: x^{*} x=1, x \in \mathbf{C}^{n}\right\} .
$$

$W(A)$ is a compact convex subset of $\mathbf{C}$, and if $A$ is hermitian then $W(A)$ is an interval on the real axis.

Proposition 1. $W(A)=\{\operatorname{tr} P A: P \in B\}$.

ProOF. Since the trace is a continuous linear functional and $B$ is a compact set, then $\{\operatorname{tr} P A: P \in B\}$ is a compact convex set which contains $W(A)$.

On the other hand each $P \in B$ is a convex combination of the extreme points of $B$. Since $B$ is a cross section of $P_{n}$ determined by a positive linear functional, the extreme points of $B$ are the intersection of the extreme rays of $P_{n}$ with the affine hyperplane

$$
\left\{H \in \mathscr{H}_{n}:(H, I)=1\right\} .
$$

If $P$ is such an extreme point, then $P$ is a nonnegative multiple of a rank one orthogonal projection, say $P=\alpha x x^{*}$ where $x^{*} x=1$. But since $P \in B$, then $1=(P, I)=\alpha x^{*} x=\alpha$. Thus for any $Q \in B$ we have

$$
Q=\sum_{j} \alpha_{j} x_{j} x_{j}^{*}
$$

where for all $j, x_{j}^{*} x_{j}=1, \alpha_{j} \geq 0$, and $\sum \alpha_{j}=1$. Thus for any $a \in M_{n}(\mathbf{C})$,

$$
\operatorname{tr} Q A=\operatorname{tr}\left[\left(\sum_{j} \alpha_{j} x_{j} x_{j}^{*}\right) A\right]=\sum_{j} \alpha_{j} x_{j}^{*} A x_{j} \in W(A) .
$$

The equality follows.

2. The joint angular field of values. Let $A_{1}, \ldots, A_{m}$ be $n \times n$ complex matrices. The set

$$
\left\{\left(x^{*} A_{1} x, \ldots, x^{*} A_{m} x\right): x^{*} x=1\right\}
$$

is usually taken to be the joint spacial numerical range of the $A_{j}[4, \mathrm{p} .137]$. It is known [4, p. 138] that for $m \geq 2$ this set is generally not convex. In [4] it is asserted that for hermitian matrices the joint spacial numerical range is a convex subset of $\mathbf{R}^{n}$. Unfortunately, this remark is false as the example on p. 138 of [4] can be used to show. For if $A, B$ are complex matrices with Toeplitz decompositions $A=H+i K, B=L+i M$ in which $H, K, L, M$ are hermitian, then the convexity of the joint numerical range of $H, K, L, M$ in $\mathbf{R}^{4}$ entails the convexity of the joint 
numerical range of $A$ and $B$ in $\mathbf{C}^{2}$. Direct numerical examples can also be given. Motivated by Proposition 1 we define the joint field of values of $A_{1}, \ldots, A_{m}$ to be

$$
W\left(A_{1}, \ldots, A_{m}\right)=\left\{\left(\operatorname{tr} P A_{1}, \ldots, \operatorname{tr} P A_{m}\right): P \in B\right\} .
$$

This set is a compact convex set and is the convex hull of the joint numerical range mentioned previously. It is also related to the $k$-numerical ranges of Fillmore and Williams [7].

In the remainder of the paper we shall be concerned with applications to hermitian matrices, so from this point we assume $A_{1}, \ldots, A_{m} \in \nvdash_{n}$. Thus $W\left(A_{1}, \ldots, A_{m}\right)$ $\subset R^{m}$. Let $J\left(A_{1}, \ldots, A_{m}\right)$ be the cone in $\mathbf{R}^{m}$ generated by $W\left(A_{1}, \ldots, A_{m}\right)$. When $0 \notin W\left(A_{1}, \ldots, A_{m}\right)$ then (e.g. $\left[\mathbf{9}\right.$, Lemma 1]) the cone $J\left(A_{1}, \ldots, A_{m}\right)=\{\alpha v: \alpha \geq$ $\left.0, v \in W\left(A_{1}, \ldots, A_{m}\right)\right\}$ is closed. We call $J\left(A_{1}, \ldots, A_{m}\right)$ the joint angular field of values of $A_{1}, \ldots, A_{m}$. In [2] this set was defined using a different cross section $B_{1}$ of $P_{n}$. However, since $B$ and $B_{1}$ are both cross sections of $P_{n}$, it is easy to check that they give rise to the same angular field of values.

NotATION. Let $A_{1}, \ldots, A_{m} \in \mathscr{H}_{n}$. When the matrices are clear from the context, we shall write $J$ for $J\left(A_{1}, \ldots, A_{m}\right), W$ for $W\left(A_{1}, \ldots, A_{m}\right)$, and let $S=$ $\operatorname{span}\left\{A_{1}, \ldots, A_{m}\right\}$.

From [2] we have the following result.

THEOREM 2. Let $A_{1}, \ldots, A_{m} \in \mathcal{H}_{n}$ with $0 \notin W$. The extreme rays of $J$ are among the rays determined by the points $\left(x^{*} A_{1} x, \ldots, x^{*} A_{m} x\right)$ where $x^{*} x=$ $1, x \in \mathbf{C}^{n}$. Furthermore, if $\left\{A_{1}, \ldots, A_{m}\right\}$ spans $\nvdash_{n}$, then $J$ is pointed; $J$ is full iff $\left\{A_{1}, \ldots, A_{m}\right\}$ is linearly independent.

In order to discuss the extreme rays of $J$ we want them "all to be there", that is, we want $J$ to be closed. The hypothesis $0 \notin W$ in Theorem 2 will insure this. This condition will also occur in Theorem 3. However, the closure of $J$ is not needed for Theorem 6 of [2] (which is restated below as Theorem 4), and so it is not assumed in Theorem 5 either. We can refine somewhat our knowledge of the extremal structure of the angular field of values. For convenience we take $m>1$ and $n>1$.

THEOREM 3. Let $m>1, n>1$ and $A_{1}, \ldots, A_{m} \in \mathcal{H}_{n}$. Then $0 \notin W$ iff $S \cap \operatorname{int} P_{n} \neq \emptyset$. Furthermore, if $S=\nvdash_{n}$, then every vector of the form $\left(\operatorname{tr} P A_{1}, \ldots\right.$, $\left.\operatorname{tr} P A_{m}\right)$, where $P \in P_{n}$ is of rank one, is an extremal of $J$.

Proof. Let $L$ be a subspace of $\mathbf{R}^{m}$ and let $K$ be a closed pointed full cone in $\mathbf{R}^{m}$. An easy extension of the Gordan-Stiemke theorem (cf. [8]) asserts that $L \cap \operatorname{int} K \neq \emptyset$ iff $L^{\perp} \cap K^{\prime}=\{0\}$. If we take $K=P_{n}$ which is self-dual and $L=S$, then we have

$$
S \cap \text { int } P_{n} \neq \emptyset \text { iff } S^{\perp} \cap P_{n}=\{0\} .
$$

If $0 \notin W$, then clearly $S^{\perp} \cap P_{n}=\{0\}$. Conversely, if $S \cap$ int $P_{n} \notin \emptyset$, then for some real scalars $\alpha_{1}, \ldots \alpha_{m}$ we have $\sum \alpha_{j} A_{j} \in$ int $P_{n}$. But if $0 \in W$, then for some $P \in P_{n}, P \neq 0$, we have $\left(\operatorname{tr} P A_{1}, \ldots, \operatorname{tr} P A_{m}\right)=0$. But then

$$
\sum \alpha_{j} \operatorname{tr} P A_{j}=\left\langle P, \sum \alpha_{j} A_{j}\right\rangle=0
$$

which cannot happen since $\sum \alpha_{j} A_{j}$ is positive definite. 
Now suppose $S=\nvdash_{n}$. From Theorem 2 we know that every extremal is of the desired form, so it suffices to show that every vector of the form $z=$ $\left(\operatorname{tr} P A_{1}, \ldots, \operatorname{tr} P A_{m}\right)$ with rank $P=1$ is an extremal. Suppose for some $z$ as above there exist $P_{1}, P_{2} \in P_{n}$ with $z_{j}=\left(\operatorname{tr} P_{j} A_{1}, \ldots, \operatorname{tr} P_{j} A_{m}\right)$ and $z=z_{1}+z_{2}$. But then $P-\left(P_{1}+P_{2}\right) \in S^{\perp}=\{0\}$, so that $P=P_{1}+P_{2}$. Since $P$ is an extremal of $P_{n}$, we have $P_{j}=\alpha_{j} P, j=1,2$, where $\alpha_{j} \geq 0$. Thus $z$ must be an extremal of $J$.

EXAMPLE. Let

$$
A_{1}=\left[\begin{array}{ll}
1 & 0 \\
0 & 0
\end{array}\right] \text { and } A_{2}=\left[\begin{array}{ll}
0 & 0 \\
0 & 1
\end{array}\right]
$$

Then $J\left(A_{1}, A_{2}\right)=\left\{\left(x_{1}, x_{2}\right): x_{j} \geq 0\right\}$. Each extremal of $J$ is of the appropriate form, but $\operatorname{span}\left\{A_{1}, A_{2}\right\} \neq \mathscr{H}_{2}$.

3. Applications to $\Pi\left(P_{n}\right)$. Recall from the introduction that $\Pi\left(P_{n}\right)$ is the set of all (real) linear transformation $T$ of $\nvdash_{n}$ such that $T\left(P_{n}\right) \subset P_{n}$. Following [2 and 8] we can represent each $T \in \operatorname{Hom}\left(\not_{n}\right)$ by a tensor product. Specifically, we use $H \otimes K$ to denote the dyad rather than the Kronecker product, so that for $H, K, P \in \mathscr{H}_{n}$ we have $H \otimes K(P)=(\operatorname{tr} K P) H$. Therefore for each $T \in \operatorname{Hom}\left(\mathscr{H}_{n}\right)$, there are $H_{1}, \ldots, H_{m}, K_{1}, \ldots, K_{m} \in \mathscr{H}_{n}$ such that $T=\sum_{j} H_{j} \otimes K_{j}$, and $T \in \Pi\left(P_{n}\right)$ iff for all $P \in P_{n}$

$$
T(P)=\sum_{j}\left(\operatorname{tr} K_{j} P\right) H_{j} \in \mathcal{P}_{n} .
$$

We state from [2] the connection with the joint angular field of values.

THEOREM 4. Let $H_{1}, \ldots, H_{m}, K_{1}, \ldots, K_{m} \in \not_{n}$. Then

$$
T=\sum H_{j} \otimes K_{j} \in \Pi\left(P_{n}\right) \quad \text { iff } J\left(K_{1}, \ldots, K_{m}\right) \subset J\left(H_{1}, \ldots, H_{m}\right)^{\prime} .
$$

If $T=\sum H_{j} \otimes K_{j}$, we may renumber the matrices so that $\left\{H_{1}, \ldots H_{t}\right\}$ is a maximal linearly independent subset of $\left\{H_{1}, \ldots, H_{m}\right\}$. Then we can represent $T$ as

$$
T=\sum_{j=1}^{t} H_{j} \otimes L_{j}
$$

where the $L_{j}$ are obvious real linear combinations of the $K_{i}$ which are also hermitian matrices. Thus it is reasonable to restrict ourselves to linearly independent sets of hermitian matrices. Recall that $S=\operatorname{span}\left\{A_{1}, \ldots, A_{m}\right\}$.

THEOREM 5. Let $m>1, n>1$ and let $A_{1}, \ldots, A_{m}$ be linearly independent elements of $H_{n}$. Then there are $B_{1}, \ldots, B_{m} \in H_{n}$ not all zero such that

$$
T=\sum A_{j} \otimes B_{j} \in \Pi\left(P_{n}\right) \quad \text { iff } S \cap P_{n} \neq\{0\} .
$$

Lemma. Let $A_{1}, \ldots, A_{m} \in \mathscr{H}_{n}$. If $J\left(A_{1}, \ldots, A_{m}\right)^{\prime}=\{0\}$, then $S \cap \mathcal{P}_{n}=\{0\}$. If $A_{1}, \ldots, A_{m}$ are linearly independent and $S \cap P_{n}=\{0\}$, then $J\left(A_{1}, \ldots, A_{m}\right)^{\prime}=\{0\}$.

Discussion. For $A_{1}, \ldots, A_{m} \in \mathscr{H}_{n}$ with the corresponding $S, W, J$ and $J^{\prime}$, note that $J^{\prime}=\{0\}$ iff the closure of $J$ is $\mathbf{R}^{m}$. Since $J$ is a convex set in a finitedimensional space, this is equivalent to $J=\mathbf{R}^{m}$, which is equivalent to $0 \in \operatorname{int} W$. 
Thus the hypotheses of the Lemma are not exactly the same as those of Theorem 3 where we are concerned with $S \cap \operatorname{int} P_{n}$.

PROOF.

$$
\begin{aligned}
\left(y_{1}, \ldots, y_{m}\right) \in J\left(A_{1}, \ldots, A_{m}\right)^{\prime} & \Leftrightarrow y_{1} \operatorname{tr} P A_{1}+\cdots+y_{m} \operatorname{tr} P A_{m} \geq 0 \quad \forall P \in \mathcal{P}_{n} \\
& \Leftrightarrow \operatorname{tr}\left[P\left(y_{1} A_{1}+\cdots+y_{m} A_{m}\right)\right] \geq 0 \quad \forall P \in \mathcal{P}_{n} \\
& \Leftrightarrow y_{1} A_{1}+\cdots+y_{m} A_{m} \in S \cap \mathcal{P}_{n} .
\end{aligned}
$$

Thus if $S \cap P_{n} \neq\{0\}$, then $J\left(A_{1}, \ldots, A_{m}\right)^{\prime} \neq\{0\}$. On the other hand, if $S \cap P_{n}=\{0\}$ and $A_{1}, \ldots, A_{m}$ are linearly independent, then $y_{1} A_{1}+\cdots+y_{m} A_{m} \in \mathcal{P}_{n}$ implies $y_{1}=\cdots=y_{m}=0$, so that $J\left(A_{1}, \ldots, A_{m}\right)^{\prime}=\{0\}$.

PROOF OF THEOREM 5 . If there are $B_{1}, \ldots, B_{m}$ not all zero such that $\sum A_{j} \otimes B_{j} \in \Pi\left(P_{n}\right)$, then $J\left(B_{1}, \ldots, B_{m}\right) \neq\{0\}$, so by Theorem $4 J\left(A_{1}, \ldots, A_{m}\right)^{\prime} \neq$ $\{0\}$. Thus from the Lemma $S \cap P_{n} \neq\{0\}$.

Conversely, suppose $\sum y_{j} A_{j} \in S \cap P_{n}, \sum y_{j} A_{j} \neq 0$. Then not all the $y_{j}$ are zero. Let $B_{j}=y_{j} I$. For any $P \in B$ we have $\operatorname{tr} P=1$, so that

$$
\sum A_{j} \otimes B_{j}(P)=\sum y_{j} A_{j} \in P_{n}
$$

Since every nonzero element of $P_{n}$ is a positive multiple of some $P \in B$, the result follows.

\section{REFERENCES}

1. G. P. Barker and D. Carlson, Cones of diagonally dominant matrices, Pacific J. Math. 57 (1975), $15-31$.

2. G. P. Barker, R. Hill and R. D. Haertel, On the completely positive and positive semidefinite preserving cones, Linear Algebra Appl. 56 (1984), 221-229.

3. A. Berman and A. Ben-Israel, More on linear inequalities with applications to matrix theory, J. Math. Anal. Appl. 33 (1971), 482-496.

4. F. F. Bonsall and J. Duncan, Numerical ranges. II, Cambridge Univ. Press, Cambridge, 1973.

5. M. D. Choi, Completely positive linear maps on complex matrices, Linear Algebra Appl. 10 (1975), 285-290.

6. J. de Pillis, Linear transformations which preserve hermitian and positive semidefinite operators, Pacific J. Math. 23 (1967), 129-137.

7. P. A. Fillmore and J. P. Williams, Some convexity theorems for matrices, Glasgow Math. J. 12 (1971), 110-117.

8. Ju. M. Glazman and Ju. I. Ljubic, Finite dimensional linear analysis, M.I.T. Press, Cambridge, Mass., 1974.

9. B.-S. Tam, Some results of polyhedral cones and simplicial cones, Linear and Multilinear Algebra, 4 (1977), 281-284. 64110

Department of Mathematics, University of Missouri, Kansas City, Missouri 